\title{
The exceptional set of Goldbach numbers (II)
}

by

\author{
Hongze Li (Jinan)
}

1. Introduction. A positive number which is a sum of two odd primes is called a Goldbach number. Let $E(x)$ denote the number of even numbers not exceeding $x$ which cannot be written as a sum of two odd primes. Then the Goldbach conjecture is equivalent to proving that

$$
E(x)=2 \quad \text { for every } x \geq 4 .
$$

$E(x)$ is usually called the exceptional set of Goldbach numbers. In [8] H. L. Montgomery and R. C. Vaughan proved that $E(x)=O\left(x^{1-\Delta}\right)$ for some positive constant $\Delta>0$. In [3] Chen and Pan proved that one can take $\Delta>0.01$. In [6], we proved that $E(x)=O\left(x^{0.921}\right)$. In this paper we prove the following result.

Theorem. For sufficiently large $x$,

$$
E(x)=O\left(x^{0.914}\right) .
$$

Throughout this paper, $\varepsilon$ always denotes a sufficiently small positive number that may be different at each occurrence. $A$ is assumed to be sufficiently large, $A<Y$, and $D=Y^{1+\varepsilon}$.

2. Some lemmas. Let $A<q \leq Y$ and $\chi_{q}$ be a non-principal character $\bmod q$. Write $\alpha=1-\lambda / \log D$, and assume

$$
\alpha \leq \sigma \leq 1, \quad|t| \leq D / q .
$$

Let $\chi(\bmod q)$ and $\chi_{0}(\bmod q)$ be a character and a principal character $\bmod q$, and $\mathcal{L}=\log D$.

1991 Mathematics Subject Classification: 11P32, 11P55.

This work was supported by the National Natural Science Foundation of China (Grant no 19671051). 
LemMa 1. Let $\chi$ be a non-principal character modulo $q$, and let $\phi=3 / 8$. Then for any $\varepsilon>0$ there exists a $\delta=\delta(\varepsilon)>0$ such that

$$
-\Re \frac{L^{\prime}}{L}(s, \chi) \leq-\sum_{|1+i t-\varrho| \leq \delta} \Re \frac{1}{s-\varrho}+\left(\frac{\phi}{2}+\varepsilon\right) H
$$

uniformly for

$$
1+\frac{1}{H \log H} \leq \sigma \leq 1+\frac{\log H}{H},
$$

providing that $q$ is sufficiently large; here $H=\log q(|t|+2)$.

This is Lemma 2.4 of [5].

Lemma 2. Suppose $Y$ is sufficiently large. Then no function $L(s, \chi)$ with $\chi$ primitive modulo $q \leq Y$, except for a possible exceptional one only, has a zero in the region

$$
\sigma \geq 1-\frac{0.239}{\log Y}, \quad q(|t|+1) \leq Y^{1+\varepsilon} .
$$

If the exceptional function exists, say $L(s, \widetilde{\chi})$, then $\widetilde{\chi}$ must be a real primitive character modulo $\widetilde{q} \leq Y$, and $L(s, \widetilde{\chi})$ has a real simple zero $\widetilde{\beta}$ satisfying

$$
1-\frac{0.239}{\log Y} \leq \widetilde{\beta} \leq 1-\frac{c}{\widetilde{q}^{10^{-8}}} .
$$

This is Lemma 2.3 of [6].

For a real number $a$, let $a^{*}=a \mathcal{L}^{-1}$, and let $\varrho_{j}=1-\lambda_{j}^{*}+i \gamma_{j}^{*}, j=1,2, \ldots$, denote the non-trivial zeros of $L(s, \chi)$ in $(2.1)$, with $\lambda_{j}$ in increasing order.

LEMMA 3. Suppose $\chi$ is a real non-principal character $\bmod q \leq Y$, and $\varrho_{1}$ is real. Then $\lambda_{2}>0.8$.

P r o o f. Apply Lemma 3.2 of [5].

When $\chi^{2}=\chi_{0}$ and $\varrho_{1}$ is complex, or $\chi^{3}=\chi_{0}$, we follow Lemma 9.1 of [4]. Let $a, k, \varepsilon$ be positive constants, and let $\phi=3 / 8, P(x)=x+x^{2}+\frac{2}{3} x^{3}$. Then

$$
\left(k^{2}+\frac{1}{2}\right)\left\{P\left(\frac{a+\lambda_{1}}{a}\right)-P\left(\frac{a+\lambda_{1}}{a+\lambda_{2}}\right)\right\}-2 k P(1)+\left(a+\lambda_{1}\right)(\psi+\varepsilon) \geq 0,
$$

where

$$
\psi=\frac{\phi}{2}\left(k^{2}+3 k+\frac{3}{2}\right)
$$

providing that

$$
k_{0}\left(a+\lambda_{1}\right)^{-3}+\left(a+\lambda_{2}\right)^{-3} \geq a^{-3} \quad \text { with } \quad k_{0}=\min \left(k+\frac{3}{4 k}, 4 k\right) .
$$

Taking $a=2.4, k=0.88$, we see that if $\lambda_{1} \leq 0.618$, then $\lambda_{2}>0.618$. 
Now suppose $\chi$ does not have order 2 or 3 . Let

$$
\mathcal{L}^{-1} \sum_{k} a_{k} \frac{(a+0.239)^{k}}{(k-1) !} \sum_{n=1}^{\infty} \Lambda(n) \Re\left(\frac{\chi(n)}{n^{s}}\right)\left(\frac{\log n}{\mathcal{L}}\right)^{k-1}=\Sigma(s, \chi) .
$$

Again we follow Lemma 9.1 of [4] with $a, k, \varepsilon, \phi$ and $P(x)$ as above. Then

$$
\begin{aligned}
\left(k^{2}+\frac{1}{2}\right)\left\{P\left(\frac{a+0.239}{a}\right)\right. & \left.-P\left(\frac{a+0.239}{a+\lambda_{2}}\right)\right\} \\
& -2 k P\left(\frac{a+0.239}{a+\lambda_{1}}\right)+(a+0.239)(\psi+\varepsilon) \geq 0 .
\end{aligned}
$$

Taking $a=2.21, k=0.89$, we see that if $\lambda_{1} \leq 0.575$, then $\lambda_{2}>0.575$.

Now we consider $\lambda_{3}$. Our starting point is the inequality

$$
\prod_{j=1}^{3}\left(1+\Re\left(\chi(n) n^{-i \gamma_{j}^{*}}\right)\right) \geq 0 .
$$

Let $P(x)=\sum a_{k} x^{k}=x+x^{2}+\frac{2}{3} x^{3}$ and

$$
\mathcal{L}^{-1} \sum_{k} a_{k} \frac{a^{k}}{(k-1) !} \sum_{n=1}^{\infty} \Lambda(n) \Re\left(\frac{\chi(n)}{n^{s}}\right)\left(\frac{\log n}{\mathcal{L}}\right)^{k-1}=\Sigma(s, \chi) .
$$

Then

$$
\Sigma\left(\sigma, \chi_{0}\right)+\sum_{j=1}^{3} \Sigma\left(\sigma+i \gamma_{j}^{*}, \chi\right)+\frac{1}{2} \sum_{2}+\frac{1}{4} \sum_{3} \geq 0
$$

with

$$
\sum_{2}=\sum_{1 \leq j<k \leq 3}\left\{\Sigma\left(\sigma+i \gamma_{j}^{*}+i \gamma_{k}^{*}, \chi^{2}\right)+\Sigma\left(\sigma+i \gamma_{j}^{*}-i \gamma_{k}^{*}, \chi_{0}\right)\right\}
$$

and

$$
\begin{aligned}
\sum_{3}= & \Sigma\left(\sigma+i \gamma_{1}^{*}+i \gamma_{2}^{*}+i \gamma_{3}^{*}, \chi^{3}\right)+\Sigma\left(\sigma+i \gamma_{1}^{*}+i \gamma_{2}^{*}-i \gamma_{3}^{*}, \chi\right) \\
& +\Sigma\left(\sigma+i \gamma_{1}^{*}-i \gamma_{2}^{*}+i \gamma_{3}^{*}, \chi\right)+\Sigma\left(\sigma+i \gamma_{1}^{*}-i \gamma_{2}^{*}-i \gamma_{3}^{*}, \widetilde{\chi}\right)
\end{aligned}
$$

Let $s=\sigma+i t, \sigma=1+a \mathcal{L}^{-1}$. We now observe that

$$
\Re\left(P\left(\frac{a}{(s-\varrho) \mathcal{L}}\right)\right) \geq 0
$$

for all zeros $\varrho$, since $\Re P(1 / z) \geq 0$ for $\Re z \geq 1$. Moreover, if $|1+i t-\varrho| \geq \delta$, then

$$
\Re\left(P\left(\frac{a}{(s-\varrho) \mathcal{L}}\right)\right)=O\left(\mathcal{L}^{-1}\right) .
$$

Here we follow Lemma 8.3 of [4]. Thus

$$
\Sigma\left(\sigma, \chi_{0}\right) \leq P(1)+\varepsilon,
$$




$$
\begin{gathered}
\sum_{1 \leq j<k \leq 3} \Sigma\left(\sigma+i \gamma_{j}^{*}-i \gamma_{k}^{*}, \chi_{0}\right) \leq \sum_{1 \leq j<k \leq 3} \Re\left\{P\left(\frac{a}{a+i\left(\gamma_{j}-\gamma_{k}\right)}\right)\right\}+\varepsilon \\
\Sigma\left(\sigma+i \gamma_{1}^{*}, \chi\right) \leq-P\left(\frac{a}{a+\lambda_{1}}\right)-\Re\left\{P\left(\frac{a}{a+\lambda_{2}+i\left(\gamma_{1}-\gamma_{2}\right)}\right)\right\} \\
-\Re\left\{P\left(\frac{a}{a+\lambda_{3}+i\left(\gamma_{1}-\gamma_{3}\right)}\right)\right\}+a\left(\frac{\phi}{2}+\varepsilon\right) \\
\Sigma\left(\sigma+i \gamma_{2}^{*}, \chi\right) \leq-P\left(\frac{a}{a+\lambda_{2}}\right)-\Re\left\{P\left(\frac{a}{a+\lambda_{3}+i\left(\gamma_{2}-\gamma_{3}\right)}\right)\right\}+a\left(\frac{\phi}{2}+\varepsilon\right), \\
\Sigma\left(\sigma+i \gamma_{3}^{*}, \chi\right) \leq-P\left(\frac{a}{a+\lambda_{3}}\right)+a\left(\frac{\phi}{2}+\varepsilon\right) \\
\sum \sum_{1 \leq j<k \leq 3}\left(\sigma+i \gamma_{j}^{*}-i \gamma_{k}^{*}, \chi^{2}\right) \leq a\left(\frac{3}{2} \phi+\varepsilon\right) \\
\Sigma\left(\sigma+i \gamma_{1}^{*}+i \gamma_{2}^{*}-i \gamma_{3}^{*}, \chi\right) \leq-\Re\left\{P\left(\frac{a}{a+\lambda_{1}+i\left(\gamma_{2}-\gamma_{3}\right)}\right)\right\} \\
-\Re\left\{P\left(\frac{a}{a+\lambda_{2}+i\left(\gamma_{1}-\gamma_{3}\right)}\right)\right\}+a\left(\frac{\phi}{2}+\varepsilon\right), \\
\Sigma\left(\sigma+i \gamma_{1}^{*}-i \gamma_{2}^{*}+i \gamma_{3}^{*}, \chi\right) \leq-\Re\left\{P\left(\frac{a}{a+\lambda_{3}+i\left(\gamma_{1}-\gamma_{2}\right)}\right)\right\}+a\left(\frac{\phi}{2}+\varepsilon\right), \\
\Sigma\left(\sigma+i \gamma_{1}^{*}+i \gamma_{2}^{*}+i \gamma_{3}^{*}, \chi^{3}\right) \leq a(\phi / 2+\varepsilon), \\
\Sigma\left(\sigma+i \gamma_{1}^{*}-i \gamma_{2}^{*}-i \gamma_{3}^{*}, \tilde{\chi}\right) \leq a(\phi / 2+\varepsilon) .
\end{gathered}
$$

Hence

$$
\begin{aligned}
P(1)-P\left(\frac{a}{a+\lambda_{1}}\right) & -P\left(\frac{a}{a+\lambda_{2}}\right)-P\left(\frac{a}{a+\lambda_{3}}\right)+a\left(\frac{11}{4} \phi+\varepsilon\right) \\
& +\frac{1}{2} \Re\left\{P\left(\frac{a}{a+i\left(\gamma_{1}-\gamma_{2}\right)}\right)-2 P\left(\frac{a}{a+\lambda_{2}+i\left(\gamma_{1}-\gamma_{2}\right)}\right)\right. \\
& \left.-\frac{1}{2} P\left(\frac{a}{a+\lambda_{3}+i\left(\gamma_{1}-\gamma_{2}\right)}\right)\right\} \\
& +\frac{1}{2} \Re\left\{P\left(\frac{a}{a+i\left(\gamma_{1}-\gamma_{3}\right)}\right)-2 P\left(\frac{a}{a+\lambda_{3}+i\left(\gamma_{1}-\gamma_{3}\right)}\right)\right. \\
& \left.-\frac{1}{2} P\left(\frac{a}{a+\lambda_{2}+i\left(\gamma_{1}-\gamma_{3}\right)}\right)\right\} \\
& +\frac{1}{2} \Re\left\{P\left(\frac{a}{a+i\left(\gamma_{2}-\gamma_{3}\right)}\right)-2 P\left(\frac{a}{a+\lambda_{3}+i\left(\gamma_{2}-\gamma_{3}\right)}\right)\right. \\
& \left.-\frac{1}{2} P\left(\frac{a}{a+\lambda_{1}+i\left(\gamma_{2}-\gamma_{3}\right)}\right)\right\} \geq 0 .
\end{aligned}
$$


Providing that

$$
a^{-3} \leq \frac{5}{2}\left(a+\lambda_{3}\right)^{-3}
$$

we have

$$
P(1)-P\left(\frac{a}{a+\lambda_{1}}\right)-P\left(\frac{a}{a+\lambda_{2}}\right)-P\left(\frac{a}{a+\lambda_{3}}\right)+a\left(\frac{11}{4} \phi+\varepsilon\right) \geq 0 .
$$

Taking $a=2$, we have $\lambda_{3} \geq 0.68$.

LEMMA 4. Suppose $\chi$ is a non-principal character $\bmod q \leq Y$, and $\varrho_{1}, \varrho_{2}, \varrho_{3}$ are the zeros of $L(s, \chi)$. Then

$$
\lambda_{2}>0.575, \quad \lambda_{3}>0.618 \text {. }
$$

Lemma 5. Suppose $\chi \neq \chi_{0}$ is a character $\bmod q \leq Y$. Let $n_{0}, n_{1}, n_{2}$ denote the numbers of zeros of $L(s, \chi)$ in the rectangles

$$
\begin{aligned}
& R_{0}: 1-\mathcal{L}^{-1} \leq \sigma \leq 1,\left|t-t_{0}\right| \leq 5.8 \mathcal{L}^{-1}, \\
& R_{1}: 1-5 \mathcal{L}^{-1} \leq \sigma \leq 1,\left|t-t_{1}\right| \leq 23.4 \mathcal{L}^{-1}, \\
& R_{2}: 1-\lambda_{+} \mathcal{L}^{-1} \leq \sigma \leq 1,\left|t-t_{2}\right| \leq 23.4 \mathcal{L}^{-1},
\end{aligned}
$$

where $t_{0}, t_{1}, t_{2}$ are real numbers satisfying $\left|t_{i}\right| \leq T$, and $5<\lambda_{+} \leq \log \log \mathcal{L}$. Then

$$
n_{0} \leq 3, \quad n_{1} \leq 10, \quad n_{2} \leq 0.2292\left(\lambda_{+}+42.9\right) .
$$

Proof. It is well known that

$$
-\frac{\zeta^{\prime}}{\zeta}(\sigma)-\Re \frac{L^{\prime}}{L}(s, \chi) \geq 0
$$

here $\sigma=\Re s$.

(i) We consider the rectangle $R_{0}$. Let $s=\sigma+i t_{0}, \sigma=1+8.4 \mathcal{L}^{-1}$, and denote by $\varrho=1-\lambda^{*}+i \gamma$ the zero of $L(s, \chi)$ in $R_{0}$, hence $0 \leq \lambda \leq 1,\left|\gamma-t_{0}\right| \leq$ $5.8 \mathcal{L}^{-1}$, and

$$
-\Re \frac{1}{s-\varrho}=-\mathcal{L} \frac{8.4+\lambda}{(8.4+\lambda)^{2}+\left(\left(\gamma-t_{0}\right) \mathcal{L}\right)^{2}} \leq-\mathcal{L} \frac{9.4}{9.4^{2}+5.8^{2}} .
$$

By Lemma 1,

$$
-\Re \frac{L^{\prime}}{L}(s, \chi) \leq-\sum_{\left|1+i t_{0}-\varrho\right| \leq \delta} \Re \frac{1}{s-\varrho}+0.18751 \mathcal{L} .
$$

If $\left|1+i t_{0}-\varrho\right|>\delta$ then $\Re \frac{1}{s-\varrho}=O(1)$. So

$$
-\Re \frac{L^{\prime}}{L}(s, \chi) \leq \mathcal{L}\left(0.18751-\frac{9.4 n_{0}}{9.4^{2}+5.8^{2}}\right) .
$$

Since $-\frac{\zeta^{\prime}}{\zeta}(\sigma) \leq \frac{1}{\sigma-1}+A$, where $A$ is an absolute constant, we have

$$
\frac{9.4 n_{0}}{9.4^{2}+5.8^{2}} \leq \frac{1}{8.4}+0.18752, \quad n_{0} \leq 3
$$


(ii) The rectangles $R_{1}$ and $R_{2}$ are treated as $R_{0}$ in (i) but with $\sigma=$ $1+24 \mathcal{L}^{-1}$. Thus $n_{1} \leq 10, n_{2} \leq 0.2292\left(\lambda_{+}+42.9\right)$.

3. The zero density estimate of the Dirichlet $L$-function near the line $\sigma=1$. Let $A<q \leq Y$ and $\chi_{q}$ be a non-principal character $\bmod q$. Write $\alpha=1-\lambda / \log D$, and assume

$$
\alpha \leq \sigma \leq 1, \quad|t| \leq D / q .
$$

Let $S_{j q}=\left\{\chi_{q}: L\left(s, \chi_{q}\right)\right.$ has only $j$ zeros in the region (3.1)\}. Suppose $A<q_{0} \leq Y$ and define

$$
\begin{aligned}
& N_{1}^{*}(\alpha, Y)=N_{1}^{*}(\lambda, Y)=\sum_{\substack{A<q \leq Y \\
\left[q, q_{0}\right] \leq D^{\varepsilon}\left(q, q_{0}\right)}} \sum_{j \geq 1} \sum_{\chi \in S_{j q}}^{*} j, \\
& N^{*}(\alpha, Y)=N^{*}(\lambda, Y)=\sum_{A<q \leq Y} \sum_{j \geq 1} \sum_{\chi \in S_{j q}}^{*} j,
\end{aligned}
$$

where $\sum^{*}$ indicates that the sum is over primitive characters. In this section we will prove the following lemma which improves Lemma 2.1 of [6].

Lemma 6. Suppose $A<q_{0} \leq Y$ and $0<\lambda \leq \varepsilon \log D$. Then

$$
\begin{aligned}
& N_{1}^{*}(\alpha, Y)=N_{1}^{*}(\lambda, Y) \leq \begin{cases}4.356 C_{1}(\lambda) e^{4.064 \lambda}, & 0.517<\lambda \leq 0.575, \\
8.46 C_{2}(\lambda) e^{4.12 \lambda}, & 0.575<\lambda \leq 0.618, \\
14.3 C_{3}(\lambda) e^{4.5 \lambda}, & 0.618<\lambda \leq 1, \\
104.1 C_{4}(\lambda) e^{3.42 \lambda}, & 1<\lambda \leq 5, \\
268.6 e^{2.16 \lambda}, & 5<\lambda \leq \varepsilon \log D,\end{cases} \\
& N^{*}(\alpha, Y)=N^{*}(\lambda, Y) \leq \begin{cases}3.632 C_{5}(\lambda) e^{5.2 \lambda}, & 0.334<\lambda \leq 0.517, \\
4.338 C_{6}(\lambda) e^{4.82 \lambda}, & 0.517<\lambda \leq 0.575, \\
10.42 C_{7}(\lambda) e^{4.5 \lambda}, & 0.575<\lambda \leq 0.618, \\
14.91 C_{8}(\lambda) e^{5.2 \lambda}, & 0.618<\lambda \leq 1, \\
104.8 C_{9}(\lambda) e^{4.16 \lambda}, & 1<\lambda \leq 5, \\
279.7 e^{2.9 \lambda}, & 5<\lambda \leq \varepsilon \log D,\end{cases}
\end{aligned}
$$

where

$$
\begin{aligned}
& C_{1}(\lambda)=\lambda^{-1}\left(1-e^{-4.064 \lambda} \frac{e^{2.808 \lambda}-e^{1.76 \lambda}}{1.048 \lambda}\right), \\
& C_{2}(\lambda)=\lambda^{-1}\left(1-e^{-4.12 \lambda} \frac{e^{2.855 \lambda}-e^{1.78 \lambda}}{1.075 \lambda}\right), \\
& C_{3}(\lambda)=\lambda^{-1}\left(1-e^{-4.5 \lambda} \frac{e^{3.198 \lambda}-e^{2.013 \lambda}}{1.185 \lambda}\right), \\
& C_{4}(\lambda)=\lambda^{-1}\left(1-e^{-3.42 \lambda} \frac{e^{2.358 \lambda}-e^{1.64 \lambda}}{0.718 \lambda}\right),
\end{aligned}
$$




$$
\begin{aligned}
& C_{5}(\lambda)=\lambda^{-1}\left(1-e^{-5.2 \lambda} \frac{e^{3.866 \lambda}-e^{2.668 \lambda}}{1.198 \lambda}\right), \\
& C_{6}(\lambda)=\lambda^{-1}\left(1-e^{-4.82 \lambda} \frac{e^{3.565 \lambda}-e^{2.51 \lambda}}{1.055 \lambda}\right), \\
& C_{7}(\lambda)=\lambda^{-1}\left(1-e^{-4.5 \lambda} \frac{e^{3.32 \lambda}-e^{2.36 \lambda}}{0.96 \lambda}\right), \\
& C_{8}(\lambda)=\lambda^{-1}\left(1-e^{-5.2 \lambda} \frac{e^{3.928 \lambda}-e^{2.7312 \lambda}}{1.1968 \lambda}\right), \\
& C_{9}(\lambda)=\lambda^{-1}\left(1-e^{-4.16 \lambda} \frac{e^{3.104 \lambda}-e^{2.38 \lambda}}{0.724 \lambda}\right) .
\end{aligned}
$$

Proof. We use the method of Section 3 of [7]. For $1 \leq j \leq 4$, let $h_{j}$ denote positive constants which satisfy

$$
h_{1}<h_{2}<h_{3}, \quad h_{2}+h_{4}+3 / 8<h_{3}, \quad 2 h_{4}+3 / 8<h_{1}
$$

when we consider $N_{1}^{*}(\alpha, Y)$, and

$$
h_{1}<h_{2}<h_{3}, \quad h_{2}+h_{4}+3 / 8<h_{3}, \quad 2 h_{4}+3 / 4<h_{1}
$$

when we consider $N^{*}(\alpha, Y)$.

Let

$$
z_{j}:=D^{h_{j}}, \quad \alpha:=1-\lambda \mathcal{L}^{-1}, \quad \lambda \leq \varepsilon \mathcal{L} .
$$

For positive $\delta_{1}, \delta_{3}$, let

$$
\begin{aligned}
\kappa(s):= & s^{-2}\left\{\left(e^{-\left(1-\delta_{1}\right)\left(\log z_{1}\right) s}-e^{-\left(\log z_{1}\right) s}\right) \delta_{3}\left(\log z_{3}\right)\right. \\
& \left.-\left(e^{-\left(\log z_{3}\right) s}-e^{-\left(1+\delta_{3}\right)\left(\log z_{3}\right) s}\right) \delta_{1}\left(\log z_{1}\right)\right\} .
\end{aligned}
$$

For a zero $\varrho_{0} \in D$, let

$$
M\left(\varrho_{0}\right):=\sum_{\varrho(\chi)}\left|\kappa\left(\varrho(\chi)+\bar{\varrho}_{0}-2 \alpha\right)\right|,
$$

where the sum is over the zeros of $L(s, \chi)$ in (3.1). Then if $2 h_{4}+3 / 8<$ $\left(1-\delta_{1}\right) h_{1}$, then as in (3.17) of [7] we have

$$
\begin{aligned}
N_{1}^{*}(\alpha, Y) \leq & \frac{(1+\delta) \max _{\varrho_{0}} M\left(\varrho_{0}\right)}{2(1-\alpha)\left(h_{2}-h_{1}\right) \delta_{1} \delta_{3} h_{1} h_{3} h_{4} \mathcal{L}^{4}} \\
& \times\left(D^{2 h_{3}(1-\alpha)}-\frac{(2 \alpha-1)\left(D^{2 h_{2}(1-\alpha)}-D^{2 h_{1}(1-\alpha)}\right)}{2(1-\alpha)\left(h_{2}-h_{1}\right) \mathcal{L}}\right) \\
\leq & \frac{(1+\delta) \max _{\varrho_{0}} M\left(\varrho_{0}\right)}{2 \lambda\left(h_{2}-h_{1}\right) \delta_{1} \delta_{3} h_{1} h_{3} h_{4} \mathcal{L}^{3}}\left(e^{2 h_{3} \lambda}-\frac{e^{2 h_{2} \lambda}-e^{2 h_{1} \lambda}}{2 \lambda\left(h_{2}-h_{1}\right)}\right) .
\end{aligned}
$$


If $2 h_{4}+3 / 4<\left(1-\delta_{1}\right) h_{1}$, then as in (3.17) of [7] we have

$$
\begin{aligned}
N^{*}(\alpha, Y) \leq & \frac{(1+\delta) \max _{\varrho_{0}} M\left(\varrho_{0}\right)}{2(1-\alpha)\left(h_{2}-h_{1}\right) \delta_{1} \delta_{3} h_{1} h_{3} h_{4} \mathcal{L}^{4}} \\
& \times\left(D^{2 h_{3}(1-\alpha)}-\frac{(2 \alpha-1)\left(D^{2 h_{2}(1-\alpha)}-D^{2 h_{1}(1-\alpha)}\right)}{2(1-\alpha)\left(h_{2}-h_{1}\right) \mathcal{L}}\right) \\
\leq & \frac{(1+\delta) \max _{\varrho_{0}} M\left(\varrho_{0}\right)}{2 \lambda\left(h_{2}-h_{1}\right) \delta_{1} \delta_{3} h_{1} h_{3} h_{4} \mathcal{L}^{3}}\left(e^{2 h_{3} \lambda}-\frac{e^{2 h_{2} \lambda}-e^{2 h_{1} \lambda}}{2 \lambda\left(h_{2}-h_{1}\right)}\right) .
\end{aligned}
$$

(i) If $5<\lambda \leq \varepsilon \mathcal{L}$, let $\Delta=23.4 \mathcal{L}^{-1}$. As in [7], by Lemma 5 we have

$$
\begin{aligned}
M\left(\varrho_{0}\right) \leq & 0.2292(\lambda+42.9) \mathcal{L}^{3}(1 / 2) \\
& \times\left\{\left(\delta_{1} h_{1}\left(2 \delta_{3}+\delta_{3}^{2}\right) h_{3}^{2}-\delta_{3} h_{3}\left(2 \delta_{1}-\delta_{1}^{2}\right) h_{1}^{2}\right)\right. \\
& \left.+(\pi / 23.4)^{2}\left(\delta_{1} h_{1}+\delta_{3} h_{3}\right)\right\} .
\end{aligned}
$$

Choose $h_{1}=0.58, h_{2}=0.669, h_{3}=1.08, h_{4}=0.0353, \delta_{1} h_{1}=\delta_{3} h_{3}=$ $\pi / 23.4$. By (3.9) we have

$$
N_{1}^{*}(\alpha, Y) \leq 268.6 e^{2.16 \lambda} .
$$

Choose $h_{1}=0.95, h_{2}=1.042, h_{3}=1.45, h_{4}=0.0328, \delta_{1} h_{1}=\delta_{3} h_{3}=$ $\pi / 23.4$. By (3.10) we have

$$
N^{*}(\alpha, Y) \leq 279.7 e^{2.9 \lambda}
$$

(ii) If $1<\lambda \leq 5$, then as in [7], by Lemma $5\left(n_{1} \leq 10\right)$ we have

$$
\begin{aligned}
M\left(\varrho_{0}\right) \leq & (10 / 2) \mathcal{L}^{3} \\
& \times\left\{\left(\delta_{1} h_{1}\left(2 \delta_{3}+\delta_{3}^{2}\right) h_{3}^{2}-\delta_{3} h_{3}\left(2 \delta_{1}-\delta_{1}^{2}\right) h_{1}^{2}\right)\right. \\
& \left.+(\pi / 23.4)^{2}\left(\delta_{1} h_{1}+\delta_{3} h_{3}\right)\right\} .
\end{aligned}
$$

Choose $h_{1}=0.82, h_{2}=1.179, h_{3}=1.71, h_{4}=0.155, \delta_{1} h_{1}=\delta_{3} h_{3}=\pi / 23.4$. By (3.9) we have

$$
N_{1}^{*}(\alpha, Y) \leq 104.1 C_{4}(\lambda) e^{3.42 \lambda} .
$$

Choose $h_{1}=1.19, h_{2}=1.552, h_{3}=2.08, h_{4}=0.1528, \delta_{1} h_{1}=\delta_{3} h_{3}=$ $\pi / 23.4$. By (3.10) we have

$$
N^{*}(\alpha, Y) \leq 104.8 C_{9}(\lambda) e^{4.16 \lambda} .
$$

(iii) If $0.618<\lambda \leq 1$, then as in [7], by Lemma 5 we have

$$
\begin{aligned}
\left(\frac{1}{a}-\frac{1}{a+1}-\right. & \left.\frac{2(a+1)}{(a+1)^{2}+5.8^{2}}+0.1876\right) \\
& \times \max \left\{\frac{a+1}{5.8^{2}}+\frac{1}{a+1}, \frac{a+0.618}{5.8^{2}}+\frac{1}{a+0.618}\right\} \leq 0.014621 .
\end{aligned}
$$


For $a=6.3$,

$$
\begin{aligned}
M\left(\varrho_{0}\right) \leq & \left\{1.5\left(\delta_{1} h_{1}\left(2 \delta_{3}+\delta_{3}^{2}\right) h_{3}^{2}-\delta_{3} h_{3}\left(2 \delta_{1}-\delta_{1}^{2}\right) h_{1}^{2}\right)\right. \\
& \left.+2 \cdot 0.014621 \cdot\left(\delta_{1} h_{1}+\delta_{3} h_{3}\right)\right\} \mathcal{L}^{3} .
\end{aligned}
$$

Choose $h_{1}=1.0065, h_{2}=1.599, h_{3}=2.25, h_{4}=0.2759, \delta_{1}=0.079$, $\delta_{3}=0.094$. By (3.9) we have

$$
N_{1}^{*}(\alpha, Y) \leq 14.3 C_{3}(\lambda) e^{4.5 \lambda} .
$$

Choose $h_{1}=1.3656, h_{2}=1.964, h_{3}=2.6, h_{4}=0.26, \delta_{1}=0.07, \delta_{3}=0.094$. By (3.10) we have

$$
N^{*}(\alpha, Y) \leq 14.91 C_{8}(\lambda) e^{5.2 \lambda} .
$$

(iv) If $0.575<\lambda \leq 0.618$, then by Lemma 4 there are at most two zeros satisfying $\varrho=1-\beta / \mathcal{L}-i \gamma / \mathcal{L}, \beta<0.618$. Then, as in (v) of [7], when (3.4) holds we have

$$
\begin{aligned}
N_{1}^{*}(\alpha, Y) \leq & \frac{(1+\delta) \widetilde{M}}{2(1-\alpha)\left(h_{2}-h_{1}\right) h_{4} \mathcal{L}^{2}} \\
& \times\left(D^{2 h_{3}(1-\alpha)}-\frac{(2 \alpha-1)\left(D^{2 h_{2}(1-\alpha)}-D^{2 h_{1}(1-\alpha)}\right)}{2(1-\alpha)\left(h_{2}-h_{1}\right) \mathcal{L}}\right) \\
\leq & \frac{(1+\delta) \widetilde{M}}{2 \lambda\left(h_{2}-h_{1}\right) h_{4} \mathcal{L}}\left(e^{2 h_{3} \lambda}-\frac{e^{2 h_{2} \lambda}-e^{2 h_{1} \lambda}}{2 \lambda\left(h_{2}-h_{1}\right)}\right) .
\end{aligned}
$$

Similarly, when (3.5) holds we have

$$
N^{*}(\alpha, Y) \leq \frac{(1+\delta) \widetilde{M}}{2 \lambda\left(h_{2}-h_{1}\right) h_{4} \mathcal{L}}\left(e^{2 h_{3} \lambda}-\frac{e^{2 h_{2} \lambda}-e^{2 h_{1} \lambda}}{2 \lambda\left(h_{2}-h_{1}\right)}\right)
$$

where

We have

$$
\widetilde{M}:=\max _{\chi \bmod q} \max _{q \leq Y} \frac{1}{1 \leq j \leq 2} \int_{\log z_{1}}^{\log z_{3}}\left|\sum_{l=1}^{j} e^{-(\varrho(l, \chi)-\alpha) x}\right|^{2} d x .
$$

$$
\begin{array}{r}
\int_{\log z_{1}}^{\log z_{3}}\left|e^{-(\varrho(\chi)-\alpha) x}\right|^{2} d x \leq\left(h_{3}-h_{1}\right) \mathcal{L}, \\
\frac{1}{2} \int_{\log z_{1}}^{\log z_{3}}\left|\sum_{l=1}^{2} e^{-(\varrho(l, \chi)-\alpha) x}\right|^{2} d x \leq 2\left(h_{3}-h_{1}\right) \mathcal{L} .
\end{array}
$$

Choose $h_{1}=0.89, h_{2}=1.4275, h_{3}=2.06, h_{4}=0.2574$. By (3.11) we have

$$
N_{1}^{*}(\alpha, Y) \leq 8.46 C_{2}(\lambda) e^{4.12 \lambda} \text {. }
$$

Choose $h_{1}=1.18, h_{2}=1.66, h_{3}=2.25, h_{4}=0.214$. By (3.12) we have

$$
N^{*}(\alpha, Y) \leq 10.42 C_{7}(\lambda) e^{4.5 \lambda} \text {. }
$$


(v) If $0.517<\lambda \leq 0.575$, then by Lemma 4 there is at most one zero satisfying $\varrho=1-\beta / \mathcal{L}-i \gamma / \mathcal{L}, \beta<0.575$. Then, as in (v) of [7], when (3.4) holds we have

$$
\begin{aligned}
N_{1}^{*}(\alpha, Y) \leq & \frac{(1+\delta)\left(h_{3}-h_{1}\right)}{2(1-\alpha)\left(h_{2}-h_{1}\right) h_{4} \mathcal{L}} \\
& \times\left(D^{2 h_{3}(1-\alpha)}-\frac{(2 \alpha-1)\left(D^{2 h_{2}(1-\alpha)}-D^{2 h_{1}(1-\alpha)}\right)}{2(1-\alpha)\left(h_{2}-h_{1}\right) \mathcal{L}}\right) \\
\leq & \frac{(1+\delta)\left(h_{3}-h_{1}\right)}{2 \lambda\left(h_{2}-h_{1}\right) h_{4}}\left(e^{2 h_{3} \lambda}-\frac{e^{2 h_{2} \lambda}-e^{2 h_{1} \lambda}}{2 \lambda\left(h_{2}-h_{1}\right)}\right) .
\end{aligned}
$$

When (3.5) holds we have

$$
N^{*}(\alpha, Y) \leq \frac{(1+\delta)\left(h_{3}-h_{1}\right)}{2 \lambda\left(h_{2}-h_{1}\right) h_{4}}\left(e^{2 h_{3} \lambda}-\frac{e^{2 h_{2} \lambda}-e^{2 h_{1} \lambda}}{2 \lambda\left(h_{2}-h_{1}\right)}\right) .
$$

Choose $h_{1}=0.88, h_{2}=1.404, h_{3}=2.032, h_{4}=0.2524$. By (3.13) we have

$$
N_{1}^{*}(\alpha, Y) \leq 4.356 C_{1}(\lambda) e^{4.064 \lambda} .
$$

Choose $h_{1}=1.255, h_{2}=1.7825, h_{3}=2.41, h_{4}=0.2524$. By (3.14) we have

$$
N^{*}(\alpha, Y) \leq 4.338 C_{6}(\lambda) e^{4.82 \lambda} .
$$

(vi) If $0.334<\lambda \leq 0.517$, then as above, when (3.5) holds we have

$$
N^{*}(\alpha, Y) \leq \frac{(1+\delta)\left(h_{3}-h_{1}\right)}{2 \lambda\left(h_{2}-h_{1}\right) h_{4}}\left(e^{2 h_{3} \lambda}-\frac{e^{2 h_{2} \lambda}-e^{2 h_{1} \lambda}}{2 \lambda\left(h_{2}-h_{1}\right)}\right) .
$$

Choose $h_{1}=1.334, h_{2}=1.933, h_{3}=2.6, h_{4}=0.291$. By (3.15) we have

$$
N^{*}(\alpha, Y) \leq 3.632 C_{5}(\lambda) e^{5.2 \lambda} \text {. }
$$

If $q_{1}, q_{2} \leq Y$, we consider the zeros of $L\left(s, \chi_{q_{1}}\right)$ and $L\left(s, \chi_{q_{2}}\right)$ for nonprincipal characters $\chi_{q_{1}}$ and $\chi_{q_{2}}$. If $\varrho_{1}=\beta_{1}+i \gamma_{1}=1-\lambda_{1} / \log Y+i \gamma_{1}$ is a zero of $L\left(s, \chi_{q_{1}}\right)$ satisfying $q_{1}\left(\left|\gamma_{1}\right|+1\right) \leq Y^{1+\varepsilon}$ and $\varrho_{2}=\beta_{2}+i \gamma_{2}=$ $1-\lambda_{2} / \log Y+i \gamma_{2}$ is a zero of $L\left(s, \chi_{q_{2}}\right)$ satisfying $q_{2}\left(\left|\gamma_{2}\right|+1\right) \leq Y^{1+\varepsilon}$, then we have the lower bounds for $\lambda_{2}$ given in Table 1. If $\left[q_{1}, q_{2}\right] \leq Y^{\varepsilon}\left(q_{1}, q_{2}\right)$, then we have the lower bounds for $\lambda_{2}$ given in Table 2 .

Table 1. The lower bounds for $\lambda_{2}$

\begin{tabular}{cl}
\hline$\lambda_{1}$ & \multicolumn{1}{c}{$\lambda_{2}$} \\
\hline 0.24 & 0.444 \\
0.26 & 0.418 \\
0.28 & 0.393 \\
0.30 & 0.37 \\
0.32 & 0.349 \\
0.334 & 0.334 \\
\hline
\end{tabular}

Table 2. The lower bounds for $\lambda_{2}$

\begin{tabular}{cc|cc}
\hline$\lambda_{1}$ & \multicolumn{1}{|c|}{$\lambda_{2}$} & \multicolumn{1}{c}{$\lambda_{1}$} & \multicolumn{1}{c}{$\lambda_{2}$} \\
\hline 0.22 & 1.189 & 0.38 & 0.745 \\
0.24 & 1.116 & 0.40 & 0.706 \\
0.26 & 1.050 & 0.42 & 0.669 \\
0.28 & 0.989 & 0.44 & 0.634 \\
0.30 & 0.933 & 0.46 & 0.601 \\
0.32 & 0.881 & 0.48 & 0.570 \\
0.34 & 0.832 & 0.50 & 0.541 \\
0.36 & 0.787 & 0.517 & 0.517 \\
\hline
\end{tabular}


In each table, following the convention of [6], the entries indicate that if $\lambda_{1}$ does not exceed the first entry, then $\lambda_{2}$ is no smaller than the second entry.

4. The circle method. Suppose $x$ is a sufficiently large positive number, and $Y=x^{\lambda}$ where $\lambda=0.0862$. Let

$$
S(\alpha)=\sum_{Y<p \leq x} \log p e(\alpha p), \quad D(n)=D(n ; x, Y)=\sum_{\substack{n=p_{1}+p_{2} \\ Y<p_{1}, p_{2} \leq x}} \log p_{1} \log p_{2} .
$$

Then

$$
D(n)=\int_{0}^{1} S^{2}(\alpha) e(-\alpha n) d \alpha
$$

Trivially, $D(n)=0$ if $n \leq 2 Y$ or $n>2 x$, and $n$ is a Goldbach number if $D(n)>0$.

Let $Q=x^{1-\lambda}, \tau=Q^{-1}$ and

$$
E_{1}=\bigcup_{1 \leq q \leq Y} \bigcup_{\substack{1 \leq a \leq q \\(a, q)=1}} I(a, q), \quad E_{2}=(-\tau, 1-\tau] \backslash E_{1}
$$

where

$$
I(a, q)=\left[\frac{a}{q}-\frac{1}{q Q}, \frac{a}{q}+\frac{1}{q Q}\right] .
$$

Then

$$
\begin{aligned}
D(n) & =\int_{-\tau}^{1-\tau} S^{2}(\alpha) e(-\alpha n) d \alpha \\
& =\int_{E_{1}} S^{2}(\alpha) e(-\alpha n) d \alpha+\int_{E_{2}} S^{2}(\alpha) e(-\alpha n) d \alpha \\
& =D_{1}(n)+D_{2}(n) .
\end{aligned}
$$

Lemma 7. Let $M(x)$ denote the number of integers $n \in[(1-\varepsilon) x, x]$ for which

$$
\left|D_{2}(n)\right|>0.5 x^{1-10^{-5} \lambda} .
$$

Then

$$
M(x) \ll x^{1-\left(1-10^{-4}\right) \lambda} .
$$

Pro of. Apply Lemma 8 of [3].

Now we consider the integral on the major arcs. For $\alpha \in I(a, q) \subset E_{1}$, we write $\alpha=a / q+\theta,(a, q)=1, q \leq Y,|\theta| \leq 1 /(q Q)$. Moreover, suppose that $\widetilde{q}$, $\widetilde{\chi}$ and $\widetilde{\beta}$ are the possible modulus, primitive character and zero respectively, 
with $\widetilde{q} \leq Y$. Let

$$
\begin{aligned}
T(\theta) & =\sum_{Y<m \leq x} e(m \theta), \\
\widetilde{T}(\theta) & =-\sum_{Y<m \leq x} m^{\widetilde{\beta}-1} e(m \theta), \\
\widehat{S}(\theta, \chi) & =\sum_{Y<p \leq x} \chi(p) \log p e(p \theta),
\end{aligned}
$$

$\chi$ being a character modulo $q, q \leq Y$, and

$$
\left\{\begin{aligned}
\widehat{S}\left(\theta, \chi_{q}^{0}\right) & =T(\theta)+W\left(\theta, \chi_{q}^{0}\right), & & \\
\widehat{S}\left(\theta, \chi_{q}^{0} \widetilde{\chi}\right) & =\widetilde{T}(\theta)+W\left(\theta, \chi_{q}^{0} \widetilde{\chi}\right) & & \text { if } \widetilde{q} \mid q, \\
\widehat{S}\left(\theta, \chi_{q}\right) & =W\left(\theta, \chi_{q}\right) & & \text { otherwise. }
\end{aligned}\right.
$$

Then if the exceptional character exists we have

$$
D_{1}(n)=\sum_{q \leq Y} \sum_{\substack{a \leq q \\(a, q)=1}} \int_{a / q-1 /(q Q)}^{a / q+1 /(q Q)} S^{2}(\alpha) e(-\alpha n) d \alpha=\sum_{j=1}^{6} D_{1 j}(n) .
$$

Otherwise we have

$$
D_{1}(n)=\sum_{j=1}^{3} D_{1 j}(n)
$$

For the definitions of $D_{1 j}(n)$, see [3]. By the method of [8] one has

$$
\begin{aligned}
& D_{11}(n)=n C(n)+O\left(x^{1+\varepsilon} Y^{-1}\right), \\
& D_{14}(n)=\widetilde{C}(n) \widetilde{I}(n)+O\left((n, \widetilde{q}) x^{1+\varepsilon} Y^{-1}\right),
\end{aligned}
$$

where

$$
\begin{aligned}
& C(n)=\sum_{q=1}^{\infty} \frac{\mu^{2}(q)}{\phi^{2}(q)} C_{q}(-n)=\frac{n}{\phi(n)} \prod_{p \nmid n}\left(1-\frac{1}{(p-1)^{2}}\right) \\
& \widetilde{C}(n)=\sum_{\substack{q=1 \\
\widetilde{q} \mid q}}^{\infty} \frac{\tau^{2}\left(\chi_{q}^{0} \widetilde{\chi}\right)}{\phi^{2}(q)} C_{q}(-n)=\widetilde{\chi}(-1) \mu\left(\frac{\widetilde{q}}{(\widetilde{q}, n)}\right) \prod_{\substack{p \mid \widetilde{q} \\
p \nmid n}}\left(\frac{1}{p-2}\right) C(n), \\
& \widetilde{I}(n)=\sum_{Y<m \leq n-Y}(m(n-m))^{\widetilde{\beta}-1} \leq x^{(1-\varepsilon)(\widetilde{\beta}-1)} n^{\widetilde{\beta}},
\end{aligned}
$$

with

$$
\tau(\chi)=\sum_{h=1}^{q} \chi(h) e\left(\frac{h}{q}\right), \quad C_{q}(m)=\sum_{\substack{h \leq q \\(h, q)=1}} e\left(\frac{m h}{q}\right) .
$$


Let

$$
W\left(\chi_{d}\right)=\left(\int_{-1 /(d Q)}^{1 /(d Q)}\left|W\left(\theta, \chi_{d}\right)\right|^{2} d \theta\right)^{1 / 2} .
$$

Then by (20) of [3] one has

$$
D_{12}(n) \leq \frac{n}{\phi(n)}\left\{8 x^{1 / 2} W\left(\log ^{10} x\right)+O\left(\frac{x^{1 / 2} W(Y)}{\log ^{6} x}\right)\right\},
$$

where

$$
W(Y)=\sum_{d \leq Y} \sum_{\chi_{d}}^{*} W\left(\chi_{d}\right)
$$

the $*$ denoting that the sum is over primitive characters $\chi_{d}$. We have

$$
D_{15}(n) \ll \widetilde{\chi}^{2}(n) \frac{\widetilde{q}}{\phi^{2}(\widetilde{q})} \cdot \frac{n}{\phi(n)} x .
$$

From $\prod_{p \geq 5}\left(1+1 /(p-1)^{2}\right) \leq 1.132$, by the method of [1], we have

$$
\begin{aligned}
D_{16}(n) & \leq 4.1594 \frac{n}{\phi(n)} x^{1 / 2} W(Y, \widetilde{q})+\frac{n}{\phi(n)} W(Y) x^{(1-\varepsilon) / 2}, \\
D_{13}(n) & \leq 2.0797 \frac{n}{\phi(n)} W(Y) W^{\prime}(Y),
\end{aligned}
$$

where

$$
\begin{aligned}
W(Y, \widetilde{q}) & =\sum_{\substack{d \leq Y \\
[d, \widetilde{q}] \leq x^{\varepsilon}(d, \widetilde{q})}} \sum_{\chi_{d}}^{*} W\left(\chi_{d}\right), \\
W^{\prime}(Y) & =\max \sum_{\substack{d \leq Y \\
\left[d_{1}, d\right] \leq x^{\varepsilon}\left(d_{1}, d\right)}} \sum_{\chi_{d}}^{*} W\left(\chi_{d}\right) .
\end{aligned}
$$

Here the max is over $A<d_{1} \leq Y$.

5. The estimation of $W^{\prime}(Y), W(Y)$ and $W(Y, \widetilde{q})$. By Section III of [2] we have

$$
\text { (5.1) } \begin{aligned}
W\left(\chi_{d}\right) \leq & \left(1+2 \cdot 10^{-5}\right) x^{1 / 2} \sum_{\substack{\beta \geq 1 / 4 \\
\left|\gamma_{\chi_{d}}\right| \leq Y^{1+\varepsilon} d^{-1}}}^{\prime} x^{(1-\varepsilon)(\beta-1)} \\
& +O\left(x^{1 / 2-\varepsilon} \sum_{\substack{\beta \geq 1 / 4 \\
\beta \geq Y^{1.01} d^{-1}}}^{\beta-1}\right) \\
& +O\left(x^{1 / 2-0.01 \lambda} \sum_{\substack{\beta \geq 1 / 4 \\
\left|\gamma_{\chi_{d}}\right| \leq Y^{2.01}}}^{\prime} x^{\beta-1}\right)+O\left(x^{1 / 2-1.01 \lambda+\varepsilon} d^{-1}\right),
\end{aligned}
$$


where $\sum^{\prime}$ indicates that the sum does not contain the exceptional zero $\widetilde{\beta}$.

By the same methods as in [1] we have

$$
\begin{gathered}
\sum_{\substack{d \leq Y \\
\left[d_{1}, d\right] \leq Y^{\varepsilon}\left(d_{1}, d\right)}} \sum_{\chi_{d}}^{*} \sum_{\substack{\beta \geq 1 / 4 \\
\left|\gamma_{d}\right| \leq Y^{2.01}}}^{\prime} x^{\beta-1} \ll x^{0.7 \varepsilon}, \\
\sum_{d \leq Y} \sum_{\chi_{d}}^{*} \sum_{\substack{\beta \geq 1 / 4 \\
\left|\gamma_{d}\right| \leq Y^{2.01}}}^{\prime} x^{\beta-1} \ll x^{0.7 \varepsilon} .
\end{gathered}
$$

Let

$$
\begin{aligned}
I_{1} & =\sum_{\substack{d \leq Y \\
\left[d_{1}, d\right] \leq Y^{\varepsilon}\left(d_{1}, d\right)}} \sum_{\chi_{d}}^{*} \sum_{\substack{\beta \geq 1 / 4 \\
\left|\gamma_{\chi_{d}}\right| \leq Y^{1+\varepsilon} d^{-1}}}^{\prime} x^{(1-\varepsilon)(\beta-1)}, \\
I_{2} & =\sum_{d \leq Y} \sum_{\chi_{d}}^{*} \sum_{\substack{\beta \geq 1 / 4 \\
\left|\gamma_{\chi_{d}}\right| \leq Y^{1+\varepsilon} d^{-1}}}^{\prime(1-\varepsilon)(\beta-1)} .
\end{aligned}
$$

Suppose $\varrho_{\chi_{d}}=\beta_{\chi_{d}}+i \gamma_{\chi_{d}},\left|\gamma_{\chi_{d}}\right| \leq Y^{1+\varepsilon} d^{-1}$, is a zero of $L\left(s, \chi_{d}\right)$. Let $\mathcal{L}=(1+\varepsilon) \log Y$.

1) If $1-0.24 / \mathcal{L} \leq \beta_{\chi_{d}} \leq 1-0.239 / \mathcal{L}$, then by Lemma 6 and Tables 1 and 2 we have

$$
\begin{aligned}
& I_{1} \leq 2 e^{-0.239 /(\lambda+\varepsilon)}+\frac{1}{\lambda+\varepsilon} \int_{1.116}^{\infty} e^{-(1-\varepsilon) t /(\lambda+\varepsilon)} N_{1}^{*}(t, Y) d t \leq 0.136, \\
& I_{2} \leq 2 e^{-0.239 /(\lambda+\varepsilon)}+\frac{1}{\lambda+\varepsilon} \int_{0.444}^{\infty} e^{-(1-\varepsilon) t /(\lambda+\varepsilon)} N^{*}(t, Y) d t \leq 1.009 .
\end{aligned}
$$

2) If $1-0.26 / \mathcal{L} \leq \beta_{\chi_{d}} \leq 1-0.24 / \mathcal{L}$, we have $I_{1} \leq 0.143, I_{2} \leq 1.098$.

3) If $1-0.28 / \mathcal{L} \leq \beta_{\chi_{d}} \leq 1-0.26 / \mathcal{L}$, we have $I_{1} \leq 0.129, I_{2} \leq 1.177$.

4) If $1-0.30 / \mathcal{L} \leq \beta_{\chi_{d}} \leq 1-0.28 / \mathcal{L}$, we have $I_{1} \leq 0.118, I_{2} \leq 1.271$.

5) If $1-0.32 / \mathcal{L} \leq \beta_{\chi_{d}} \leq 1-0.30 / \mathcal{L}$, we have $I_{1} \leq 0.114, I_{2} \leq 1.377$.

6) If $1-0.34 / \mathcal{L} \leq \beta_{\chi_{d}} \leq 1-0.32 / \mathcal{L}$, we have $I_{1} \leq 0.118, I_{2} \leq 1.464$.

7) If $1-0.36 / \mathcal{L} \leq \beta_{\chi_{d}} \leq 1-0.34 / \mathcal{L}$, we have $I_{1} \leq 0.131, I_{2} \leq 1.374$.

8) If $1-0.38 / \mathcal{L} \leq \beta_{\chi_{d}} \leq 1-0.36 / \mathcal{L}$, we have $I_{1} \leq 0.153, I_{2} \leq 1.249$.

9) If $1-0.40 / \mathcal{L} \leq \beta_{\chi_{d}} \leq 1-0.38 / \mathcal{L}$, we have $I_{1} \leq 0.185, I_{2} \leq 1.141$.

10) If $1-0.42 / \mathcal{L} \leq \beta_{\chi_{d}} \leq 1-0.40 / \mathcal{L}$, we have $I_{1} \leq 0.229, I_{2} \leq 1.047$.

11) If $1-0.44 / \mathcal{L} \leq \beta_{\chi_{d}} \leq 1-0.42 / \mathcal{L}$, we have $I_{1} \leq 0.287, I_{2} \leq 0.967$.

12) If $1-0.46 / \mathcal{L} \leq \beta_{\chi_{d}} \leq 1-0.44 / \mathcal{L}$, we have $I_{1} \leq 0.337, I_{2} \leq 0.897$.

13) If $1-0.48 / \mathcal{L} \leq \beta_{\chi_{d}} \leq 1-0.46 / \mathcal{L}$, we have $I_{1} \leq 0.372, I_{2} \leq 0.835$.

14) If $1-0.50 / \mathcal{L} \leq \beta_{\chi_{d}} \leq 1-0.48 / \mathcal{L}$, we have $I_{1} \leq 0.395, I_{2} \leq 0.784$.

15) If $1-0.517 / \mathcal{L} \leq \beta_{\chi_{d}} \leq 1-0.50 / \mathcal{L}$, we have $I_{1} \leq 0.420, I_{2} \leq 0.738$.

16) If $1-0.517 / \mathcal{L} \geq \beta_{\chi_{d}}$, we have $I_{1} \leq 0.414, I_{2} \leq 0.704$. 
Hence in all cases we have

$$
I_{1} I_{2} \leq 0.311 .
$$

LEMMA 8. Let $\chi_{1}$ be a real non-principal character $\bmod q, \beta_{1}=1-\delta_{1} a$ real zero of $L\left(s, \chi_{1}\right)$, $\chi$ a character $\bmod q$, and $\varrho=\beta+i \gamma=1-\delta+i \gamma$ a zero of $L(s, \chi)$ with $\delta<1 / 6, \beta \leq \beta_{1}$. Suppose that $D=q(|\gamma|+1)$ is sufficiently large, that is, $D \geq D_{0}(\varepsilon)$. Then

$$
\delta_{1} \geq(2 / 3-\varepsilon)(1-6 \delta) D^{-(3 / 2+\varepsilon) \delta /(1-6 \delta)} / \log D .
$$

This is Theorem 2 of [9].

LEMma 9. If the exceptional primitive real character $\tilde{\chi}(\bmod \widetilde{q})$ exists, and the unique exceptional zero $\widetilde{\beta}$ of $L(s, \widetilde{\chi})$ satisfies $\widetilde{\delta}(\lambda+\varepsilon) \log x \leq 0.239$ where $\widetilde{\delta}=1-\widetilde{\beta}$, let $\chi_{q}$ be a primitive character $\bmod q$, and $\varrho=\beta+i \gamma=$ $1-\delta+i \gamma$ a zero of $L\left(s, \chi_{q}\right)$ with $0<\delta<\varepsilon$. Suppose that $D_{1}=[q, \widetilde{q}](|\gamma|+1)$ is sufficiently large, that is, $D_{1} \geq D_{1}(\varepsilon)$. Then

$$
\widetilde{\delta} \geq(2 / 3-\varepsilon)(1-6 \delta) D_{1}^{-(3 / 2+\varepsilon) \delta /(1-6 \delta)} / \log D_{1} .
$$

Proof. This follows by Lemma 8 and the method of Lemma 15 of [1].

By (26) of [1] we have

$$
W\left((\log x)^{10}\right) \leq 10^{-10} x^{1 / 2}
$$

By (5.1)-(5.4) and definitions of $W(Y)$ and $W^{\prime}(Y)$ we have

$$
W(Y) W^{\prime}(Y) \leq 0.311 x \text {. }
$$

Now we suppose that the exceptional primitive real character $\widetilde{\chi}(\bmod \widetilde{q})$ exists, and the unique exceptional real zero $\widetilde{\beta}$ of $L(s, \widetilde{\chi})$ satisfies $\widetilde{\delta}(\lambda+\varepsilon) \log x \leq 0.239$ where $\widetilde{\delta}=1-\widetilde{\beta}$. In this case, as above we have

$$
W(Y, \widetilde{q}) \leq W^{\prime}(Y) \leq 0.0107 x^{1 / 2}, \quad W(Y) \leq 0.884 x^{1 / 2} .
$$

Hence we have

$$
W(Y) W^{\prime}(Y) \leq 0.0095 x .
$$

We suppose, as we may, that $\widetilde{q} \leq Y, q \leq Y,[q, \widetilde{q}] \leq x^{\varepsilon}(q, \widetilde{q})$ and $|\gamma| \leq$ $Y^{1+\varepsilon} q^{-1}$, and then we may take $D_{1}=x^{\bar{\lambda}+2 \varepsilon}$ in Lemma 9. Therefore if $\widetilde{\delta}(\lambda+\varepsilon) \log x \leq 0.005$ and $\delta \leq \varepsilon$, then we have

$$
\delta \geq \frac{3.26}{\lambda \log x}
$$

If $\widetilde{\delta}(\lambda+\varepsilon) \log x \geq 0.005, \widetilde{\delta} \geq(2 / 3-\varepsilon)\left(D_{1}^{1.501 \varepsilon} \log D_{1}\right)^{-1}, \delta \leq \varepsilon$, then as above, by Lemma 9 one has

$$
\delta \geq-\frac{\log \left(1.501 \widetilde{\delta} \log D_{1}\right)}{1.501 \log D_{1}} .
$$


By Lemma 6 we have

$$
\begin{aligned}
& \sum_{\substack{d \leq Y \\
\left[d_{1}, d\right] \leq Y^{\varepsilon}\left(d_{1}, d\right)}} \sum_{\chi_{d}}^{*} \sum_{\substack{\beta \geq 1 / 4 \\
\left|\gamma_{\chi_{d}}\right| \leq Y^{1.01} d^{-1}}}^{\prime} x^{(1-\varepsilon)(\beta-1)} \\
\leq & \frac{1}{\lambda+\varepsilon} \int_{-\left(\log \left(1.501 \widetilde{\delta} \log D_{1}\right)\right) / 1.501}^{\infty} e^{-(1-\varepsilon) t /(\lambda+\varepsilon)} N_{1}^{*}(t, Y) d t+O\left(x^{-\varepsilon}\right) \\
\leq & 10^{-8}(\widetilde{\delta} \log x)+O\left(x^{-\varepsilon}\right) .
\end{aligned}
$$

Hence

$$
W^{\prime}(Y) \leq 10^{-8}(\widetilde{\delta} \log x) x^{1 / 2}+O\left(x^{1 / 2-\varepsilon}\right) .
$$

Similarly we have

$$
W\left((\log x)^{10}\right), W(Y, \widetilde{q}) \leq 10^{-8}(\widetilde{\delta} \log x) x^{1 / 2}+O\left(x^{1 / 2-\varepsilon}\right) .
$$

If $x^{-\lambda / 10^{5}} \leq \widetilde{\delta} \leq(2 / 3-\varepsilon)\left(D_{1}^{1.501 \varepsilon} \log D_{1}\right)^{-1}$, then as above, by Lemma 9 one has

$$
W\left((\log x)^{10}\right), W(Y, \widetilde{q}), W^{\prime}(Y) \leq \varepsilon(\widetilde{\delta} \log x) x^{1 / 2}+O\left(x^{1 / 2-0.01}\right) .
$$

6. Proof of the Theorem. First of all, we suppose that there is no exceptional character. When $(1-\varepsilon) x \leq n \leq x$, by (4.8), (4.9), (4.15) and (4.19) we have

$$
\begin{aligned}
D_{1}(n) \geq & n C(n)-\frac{n}{\phi(n)}\left\{8 x^{1 / 2} W\left((\log x)^{10}\right)\right. \\
& \left.+2.0797 W(Y) W^{\prime}(Y)+O\left(\frac{x^{1 / 2} W(Y)}{(\log x)^{6}}\right)\right\}+O\left(x^{1-\lambda+\varepsilon}\right) .
\end{aligned}
$$

Since $\lambda=0.0862, \prod_{p \geq 3}\left(1-1 /(p-1)^{2}\right) \geq 0.6601$, by (5.5) and (5.6) it follows that

$$
D_{1}(n) \geq \frac{n x}{\phi(n)}\left\{\prod_{p \geq 3}\left(1-\frac{1}{(p-1)^{2}}\right)-2.0797 \cdot 0.311-10^{-9}\right\} \geq 0.01 x
$$

which proves the assertion.

Now we suppose the exceptional character occurs, and $(1-\varepsilon) x \leq n \leq x$. By Section 4 we have

$$
\begin{aligned}
D_{1}(n) \geq & n C(n)+\widetilde{I}(n) \widetilde{C}(n) \\
& -\frac{n}{\phi(n)}\left\{8 x^{1 / 2} W\left((\log x)^{10}\right)+2.0797 W(Y) W^{\prime}(Y)\right. \\
& \left.+4.1594 W(Y, \widetilde{q})+W(Y) x^{(1-\varepsilon) / 2}\right\} \\
& +O\left(\frac{x^{1 / 2} W(Y)}{(\log x)^{6}}\right)+O\left(\widetilde{\chi}^{2}(n) \frac{\widetilde{q}}{\phi^{2}(\widetilde{q})} \cdot \frac{n}{\phi(n)} x\right) \\
& +O\left(x^{1-\lambda+\varepsilon}(n, \widetilde{q})\right) .
\end{aligned}
$$


1) When $(n, \widetilde{q})=1$ or $(n, \widetilde{q}) \leq x^{\left(1-10^{-4}\right) \lambda}$ and $\prod_{p \mid \widetilde{q}, p \nmid n}(p-2) \geq 1 / \varepsilon$ we follow the argument of [1]. Thus by (5.7) and (5.8) we have

$$
\begin{array}{r}
D_{1}(n) \geq \frac{n}{\phi(n)}\left\{x \prod_{p \geq 3}\left(1-\frac{1}{(p-1)^{2}}\right)-2.0797 W(Y) W^{\prime}(Y)-10^{-8} x\right. \\
\left.-4.1594 W(Y, \widetilde{q}) x^{1 / 2}\right\} \geq 0.59 x .
\end{array}
$$

2) When $(n, \widetilde{q})>x^{\left(1-10^{-4}\right) \lambda}$ we have

$$
\sum_{\substack{n \leq x \\>x^{\left(1-10^{-4}\right) \lambda}}} 1 \leq x^{1-\left(1-10^{-4}\right) \lambda+\varepsilon} .
$$

3) When $1<(n, \widetilde{q}) \leq x^{\left(1-10^{-4}\right) \lambda}$ and $\prod_{p \mid \widetilde{q}, p \nmid n}(p-2) \leq 1 / \varepsilon$, we notice that $\widetilde{\chi}(n)=0$, and from Lemma 5.1 of $[8]$ we have $\mu(\widetilde{q} /(4, \widetilde{q}))=0$ hence $16 \nmid \widetilde{q}, p^{2} \nmid \widetilde{q}(p \geq 3)$. Since $\prod_{p \mid \widetilde{q}, p \nmid n}(p-2) \leq 1 / \varepsilon$, there exists $\widetilde{q} \leq$ $16(n, \widetilde{q}) / \varepsilon^{2} \leq x^{\left(1-10^{-4}\right) \lambda+\varepsilon}$. By (4.12) and (4.13) we have

$$
n C(n)-|\widetilde{I}(n) \widetilde{C}(n)| \geq\left(n-x^{(1-\varepsilon)(\widetilde{\beta}-1)} n^{\widetilde{\beta}}\right) C(n) .
$$

When $1-\frac{0.239}{(\lambda+\varepsilon) \log x} \leq \widetilde{\beta} \leq 1-\frac{0.005}{(\lambda+\varepsilon) \log x}$, we have

$$
x^{(1-\varepsilon)(\widetilde{\beta}-1)} n^{\widetilde{\beta}} \leq 0.8905 n .
$$

By (5.7) and (5.8) we have

$$
\begin{aligned}
D_{1}(n) \geq \frac{n x}{\phi(n)}\left\{0.1095 \prod_{p \geq 3}(\right. & \left.1-\frac{1}{(p-1)^{2}}\right)-2.0797 \cdot 0.0095 \\
& \left.-10^{-8}-4.1594 \cdot 0.0107\right\} \geq 0.007 x .
\end{aligned}
$$

When $1-\frac{0.005}{(\lambda+\varepsilon) \log x} \leq \widetilde{\beta} \leq 1-\left(\frac{2}{3}-\varepsilon\right) \frac{x^{-1.501 \varepsilon \lambda}}{\lambda \log x}$, as in (48) of [1] we have

$$
n C(n)-|\widetilde{I}(n) \widetilde{C}(n)| \geq 0.62 \frac{\widetilde{\delta} n x \log n}{\phi(n)} .
$$

By (5.12) and (5.13) we have

$$
\begin{aligned}
D_{1}(n) & \geq \frac{\widetilde{\delta} n x \log n}{\phi(n)}\left\{0.62-2.0797 \cdot 10^{-7}-(8+4.1594) \cdot 10^{-8}\right\} \\
& \geq 0.6 x^{1-\varepsilon} .
\end{aligned}
$$

When $\widetilde{\beta} \geq 1-\left(\frac{2}{3}-\varepsilon\right) \frac{x^{-1.501 \varepsilon \lambda}}{\lambda \log x}$, by $\widetilde{q} \leq x^{\lambda}$ and Lemma 2 we have

$$
x^{-10^{-5} \lambda} \leq \widetilde{\delta} \leq\left(\frac{2}{3}-\varepsilon\right) \frac{x^{-1.501 \varepsilon \lambda}}{\lambda \log x},
$$


and by (5.14) we have

$$
D_{1}(n) \geq \frac{\widetilde{\delta} n x \log n}{\phi(n)}\{0.62-20 \varepsilon\} \geq 0.6 x^{1-10^{-5} \lambda} .
$$

By (6.1)-(6.7) and Lemma 7 the assertion follows.

\section{References}

[1] J. R. Chen, The exceptional set of Goldbach numbers (II), Sci. Sinica 26 (1983), $714-731$

[2] J. R. Chen and J. M. Liu, The exceptional set of Goldbach numbers (III), Chinese Quart. J. Math. 4 (1989), 1-15.

[3] J. R. Chen and C. D. Pan, The exceptional set of Goldbach numbers, Sci. Sinica 23 (1980), 416-430.

[4] D. R. Heath-Brown, Zero-free regions for Dirichlet L-functions, and the least prime in an arithmetic progression, Proc. London Math. Soc. (3) 64 (1992), 265-338.

[5] H. Z. Li, Zero-free regions for Dirichlet L-functions, Quart. J. Math. Oxford Ser. (2) 50 (1999), 13-23.

[6] -, The exceptional set of Goldbach numbers, ibid. 50 (1999).

[7] J. Y. Liu, M. C. Liu and T. Z. W ang, The number of powers of 2 in a representation of large even integers (II), Sci. China Ser. A 41 (1998), 1255-1271.

[8] H. L. Montgomery and R. C. Vaughan, The exceptional set in Goldbach's problem, Acta Arith. 27 (1975), 353-370.

[9] W. Wang, On zero distribution of Dirichlet's L-functions, J. Shandong Univ. 21 (1986), 1-13 (in Chinese).

Department of Mathematics

Shandong University

Jinan Shandong

P.R. China

E-mail: lihz@sdu.edu.cn 